«Piety to a dead man »: les limites de la consolation dans la pratique épistolaire de Sir Kenelm Digby (Angleterre, 1633)

\title{
Paula Barros
}

\section{(2) OpenEdition Journals}

Édition électronique

URL : http://journals.openedition.org/rhetorique/527

DOI : $10.4000 /$ rhetorique.527

ISSN : 2270-6909

Éditeur

UGA Éditions/Université Grenoble Alpes

Édition imprimée

ISBN : 978-2-37747-010-5

\section{Référence électronique}

Paula Barros, « "Piety to a dead man » : les limites de la consolation dans la pratique épistolaire de Sir Kenelm Digby (Angleterre, 1633) », Exercices de rhétorique [En ligne], 9 | 2017, mis en ligne le 16 juin 2017, consulté le 12 septembre 2020. URL : http://journals.openedition.org/rhetorique/527 ; DOI : https://doi.org/10.4000/rhetorique.527

Ce document a été généré automatiquement le 12 septembre 2020.

\section{c) (ㄱ)(2)}

Les contenus de la revue Exercices de rhétorique sont mis à disposition selon les termes de la Licence Creative Commons Attribution - Pas d'Utilisation Commerciale - Partage dans les Mêmes Conditions 4.0 International. 


\title{
"Piety to a dead man » : les limites de la consolation dans la pratique épistolaire de Sir Kenelm Digby (Angleterre, 1633)
}

\author{
Paula Barros
}

«Monsieur, j'espérais vous voir ici bien avant ce jour et je vous prie de me donner ce plaisir à votre convenance, quand vos affaires vous le permettront, à moins peut-être que vous ne craigniez trouver ici compagnie si mauvaise que la charité dont vous ferez preuve en me rendant visite vous coûtera trop cher $^{1}$ ». Dans ces quelques lignes extraites d'une lettre non datée, mais écrite peu de temps après le décès de sa femme, Sir Kenelm Digby exprime son déplaisir à l'égard de son ami Thomas Gell, qui avait manifestement tardé à venir lui présenter ses condoléances. Le $1^{\text {er }}$ mai 1633 , Venetia Digby s'était éteinte dans son lit à l'âge de 33 ans. Ce décès inattendu plongea Digby dans un chagrin profond, dont témoigne la quarantaine de lettres qu'il écrivit pendant les premiers mois de son veuvage. Cette correspondance entièrement consacrée à son deuil fut recopiée par Digby lui-même ou par son secrétaire et rassemblée dans un volume relié dont la couverture porte le titre In Praise of Venetia. Elle constitue un témoignage unique pour qui s'intéresse aux discours et aux pratiques du deuil et de la consolation dans l'Europe des XVI ${ }^{\mathrm{e}}$ et XVII ${ }^{\mathrm{e}}$ siècles².

2 Comme d'autres pays de l'espace européen, l'Angleterre avait hérité de la tradition consolatoire antique, pour laquelle la prise en charge de la douleur d'autrui était un devoir d'amitiés. Dans les cercles des élites éduquées, faillir à cette obligation, comme le fit l'ami de Digby, était non seulement une entorse aux règles de la bienséance, mais un manquement grave aux valeurs éthiques et sociales : l'équilibre des relations amicales, fondé sur la réciprocité et la confiance, risquait d'en être affectét. On comprend, dès lors, pourquoi Digby s'offusque de l'absence et du silence de son ami, alors même que ses lettres répètent à l'envi qu'il est insensible à toute forme de consolation. 
Philosophe, homme de sciences, diplomate et même, à ses heures, corsaire pour le compte du roi Charles I ${ }^{\text {er }}$ d'Angleterre, Sir Kenelm Digby (1603-1665) frappa l'imaginaire de ses contemporains par son comportement inhabituel après la mort de sa femme ${ }^{5}$. Alors que les règles de civilité exigeaient la modération dans la manifestation du deuil, Digby n'hésita pas à adopter publiquement la posture du veuf inconsolable. Non seulement il passa commande auprès d'Antoine Van Dyck d'un portrait de son épouse sur son lit de mort, mais il se fit représenter lui-même sous les traits du veuf affligé, par Van Dyck et Cornelius Johnson, dans deux tableaux qui reproduisent les codes de la mélancolie et du deuil inconsolable : vêtements de couleurs sombres, cheveux décoiffés, barbe hirsute, joues creuses, regard éteint ${ }^{6}$. Par ailleurs, en rassemblant la correspondance liée au décès de sa femme dans un recueil, Digby en facilita la circulation au sein de son cercle de sociabilité et en assura la transmission aux générations futures ${ }^{7}$. Véritables équivalents textuels des portraits de deuil, ces lettres traduisent, elles aussi, le désir de Digby de témoigner de l'ampleur de son chagrin et d'en préserver le souvenir pour la postérité.

Bien que consacré à un sujet unique, ce recueil de correspondance n'en présente pas moins une certaine variété. Quelques missives, parfois assez courtes, ont pour objet de remercier un parent, un ami ou une connaissance plus éloignée d'avoir effectué une visite ou envoyé une lettre de condoléance. Parmi les pièces plus longues, nombreuses sont celles qui témoignent d'une plus grande intimité avec le destinataire, qu'il s'agisse d'amis de Digby ou de son frère, à qui il adresse près de la moitié de ses lettres. Dans la plupart de ces épîtres, Digby épanche sa peine tout en explorant les recoins de son âme. Si la rhétorique de la plainte est omniprésente, le thème de la perte se décline également sur le mode encomiastique dans des passages évoquant, quelquefois longuement, le souvenir de la défunte; ailleurs, il débouche sur des spéculations philosophiques ou métaphysiques sur les retrouvailles célestes des époux. Mais c'est le motif récurrent des limites de la consolation, inefficace ou indésirable car inadaptée à la personnalité de l'épistolier, qui forme la basse continue de ce recueil de correspondance.

De cet ensemble riche et varié, nous retenons pour la présente étude trois lettres de réponse dans lesquelles Digby exprime sa reconnaissance à l'égard de certains de ses proches qui ont cherché à le réconforter. À partir de ce corpus restreint, fortement marqué par les recommandations et les modèles proposés dans les manuels épistolaires des années 1560-1640, nous nous efforcerons de mieux cerner le discours sur les limites de la consolation, ses codifications, ses valeurs et sa signification culturelle. Parce qu'ils donnent à entendre le point de vue d'un endeuillé destinataire de lettres de consolation, ces textes nous offrent la possibilité d'engager une réflexion sur la question de la réception de ce genre de discours et nous permettront d'affiner notre compréhension de la prise en charge de la douleur d'autrui dans la culture européenne de la première modernité.

\section{Les lettres de réponse : la pratique épistolaire de Digby et ses modèles}

6 À partir du milieu du XVI siècle, les secrétaires et les manuels épistolographiques accordent, toutes rubriques confondues, une place croissante aux lettres de réponse, accentuant ainsi la dimension interactive de l'échange épistolaire ${ }^{8}$. En ce qui concerne 
plus spécifiquement le genre consolatoire, l'évolution qualitative et quantitative des lettres de réponse concernant les situations de deuil est riche en enseignements. Un inventaire rapide permet d'identifier : une traduction de la lettre de Cicéron à Sulpicius dans A Panoplie of Epistles d'Abraham Fleming (1576), où Cicéron répond à la consolation que lui avait adressée son ami après la mort de sa fille Tullia; un modèle de lettre de réponse dans The English Secretorie d'Angel Day (1586), qui était le plus influent des manuels épistolaires anglais jusque dans les années 1630 ; un autre modèle dans $A$ President for Young Pen-Men de l'anonyme M. R. (1615), et non moins de dix exemples dans The Secretary in Fashion, la traduction anglaise du Secrétaire à la mode de Jean Puget de la Serre imprimée à Londres en $1640^{\circ}$. Quant à Érasme, il n'avait pas inclus de modèle de réponse développée dans la section du De conscribendis epistolis (1522) dédiée à la lettre de consolation, mais il en avait fourni les préceptes, qu'il avait agrémentés d'un bref florilège de citations extraites de la correspondance de Cicéron ${ }^{10}$. Angel Day reprit ensuite ces conseils dans son propre manuel, dont le contenu, souvent emprunté au De conscribendis epistolis, "synthétise » selon James Daybell « l'approche érasmienne et plus largement humaniste [de l'art épistolaire] et des traditions plus anciennes ${ }^{11}$ ».

Quelles sont les règles pour composer une lettre de réponse? Angel Day souligne, dans le sillage d'Érasme, que ce type de lettre doit exprimer "la reconnaissance ou l'acceptation amicale de la bienveillance de ceux qui se sont employés à nous réconforter"; si l'épistolier le juge opportun, il peut en outre louer «la sagesse, l'érudition, la faveur, le souci et l'affection " de son correspondant; en fonction des circonstances, il peut mettre en avant les «bienfaits" de la consolation ou alors indiquer que son mal est tel que les conseils prodigués se sont avérés moins efficaces qu'ils ne l'auraient été pour «beaucoup d'autres». Dans la conclusion, il doit montrer qu'il apprécie à leur juste valeur le « discernement » de son correspondant et le « mal » qu'il s'est donné ${ }^{12}$. L'une des contraintes de la lettre de réponse consiste donc à exprimer sa reconnaissance pour un service rendu. Introduit dans l'exorde et repris dans la péroraison, ce thème est en effet obligatoire. Au cœur de la lettre, la teneur de la narratio et la confirmatio dépendent ensuite des circonstances et de l'appréciation de l'épistolier.

Or, si les réponses proposées en guise de modèle dans les différents secrétaires et manuels suivent globalement ce schéma, il est intéressant de noter qu'à une exception près, les auteurs (le plus souvent fictifs) de ces lettres ne se déclarent jamais consolés par les discours réconfortants dont ils sont les destinataires. Dans A President for Yong Pen-Men, un père qui vient d'enterrer son jeune fils répond à une lettre dont l'argument principal est la soumission à la volonté de Dieu, une nécessité qu'il accepte non sans supplier son correspondant de faire preuve d'indulgence : « autant que je le puis sans offenser Dieu, permettez-moi d'être moi-même ${ }^{13}$ ». Dans l'exemple proposé par Angel Day, l'épistolier évoque brièvement les bienfaits éphémères de la consolation, mais s'attache surtout à en montrer l'inefficacité : trois quarts de la lettre sont consacrés à ce thème. Sur les dix réponses du Secrétaire à la mode, une seule décrit sans ambiguiité la transformation effectuée par le discours de consolation ${ }^{14}$; les autres affirment toutes que la douleur éprouvée est insurmontable et ne présentent des variations que dans leur appréciation plus ou moins tolérante du discours de consolation lui-même. Dans l'une d'elles, l'épistolier affirme qu'il "souhaiteroi[t] d'estre capable de consolation " pour «[s]e pouvoir servir utilement des charitables preceptes» rappelés par son correspondant ${ }^{15}$. Une autre donne la parole à un épistolier révolté contre le 
raisonnement qui sous-tend l'un des principaux arguments du discours consolatoire : « [o]n a beau me representer que mon mal est sans remede; c'est parce qu'il n'en a point que je suis sans consolation ${ }^{16}$ ». Le réconfort, lit-on ailleurs, se puise aussi bien dans les témoignages de sympathie que dans le discours exhortatif qui constitue pourtant la finalité de toute lettre de consolation: «Il faut que j'advoüe que vostre charitable lettre m'a beaucoup consolé, de la perte que j'ay faite de mon Père, \& d'autant plus encore que vous m'avez témoigné, combien vous avez esté sensible à mon affliction ${ }^{17}$ ». Les lettres de réponse mettent ainsi à la disposition du public un réservoir de formules et d'arguments permettant de souligner l'inefficacité de la consolation, un échec qu'elles rendent patent par l'expression parfois abondante du chagrin; elles ont donc aussi partie liée avec la lettre de lamentation, et plus généralement avec la rhétorique de la plainte à l'œuvre, par exemple, dans les élégies funèbres, dont elles mobilisent les dispositifs permettant l'amplification de la douleur ${ }^{18}$.

Quand Digby prend la plume pour répondre à ses amis ou à ses proches, il s'inscrit donc dans une pratique bien établie. Assez brève, la première lettre que nous présentons ici s'adresse à sa mère ${ }^{19}$. Digby y remercie «humblement» Lady Digby de sa lettre " aimante et maternelle», qui lui a donné «tout le réconfort que peut prodiguer une main terrestre ${ }^{20} »$; il prend à cœur, poursuit-il, le conseil de sa mère de "ne s'attacher fortement à aucun objet éphémère ${ }^{21}$ » ; « les grands malheurs » qui ont marqué la vie de sa mère font de celle-ci un «modèle » qu'il fait vœu de contempler ${ }^{22}$; il demande enfin la «bénédiction » de Lady Digby, l'assurant en retour de son « devoir» et se dit pour conclure son fils «humble" et "obéissant ${ }^{23}$ ". Fidèle à la structure préconisée par Angel Day, cette lettre se caractérise par sa grande retenue. Écrite le 27 juin 1633, soit près de deux mois après le décès de Venetia Digby, elle n'exprime aucune plainte. Digby y témoigne au contraire de sa volonté de se soumettre à son sort en prenant pour exemple le comportement de sa mère dans "ses grands malheurs ", une allusion à un épisode tragique de l'histoire familiale, l'arrestation et l'exécution pour haute trahison du père de Digby, qui avait participé, en 1605 , à la conspiration des poudres, un complot ourdi par un groupe de catholiques contre le roi Jacques $\mathrm{I}^{\mathrm{er}}$.

Plusieurs lettres du recueil de correspondance sont adressées à Sir John Coke, l'un des deux principaux secrétaires d'État de Charles $\mathrm{I}^{\mathrm{er}}$, un personnage puissant donc, ami et protecteur de Digby, bien que férocement anticatholique ${ }^{24}$. Parmi elles, nous retenons une réponse à une lettre de consolation datée du 26 juillet $1633^{25}$. Digby y adopte de nouveau le plan recommandé par Angel Day, mais se montre plus prolixe qu'il ne l'avait été avec Lady Digby: cette lettre compte près de 1000 mots où la précédente en comptait à peine 200. L'épistolier insiste ici sur les liens amicaux qui l'unissent à Coke et déploie un discours explicite de résistance à la consolation. Dans l'exorde, qui occupe à lui seul un tiers de la lettre, il exprime sa gratitude à l'égard de Coke, « le meilleur ami et l'homme le plus sage [qu'il ait] jamais rencontré26»; or, explique-t-il, l'amitié et la sagesse sont complémentaires dans le devoir de consolation (" consolatory offices »); les paroles consolatrices de Coke l'ont en effet fortifié, bien plus que ne le pourraient les écrits des philosophes - il cite Sénèque, Plutarque et Boèce - qui «ne nous disent que ce que nous devons faire, mais ne peuvent guère préparer à l'avance nos affections pour mieux nous aider à digérer leurs préceptes ${ }^{27} »$; c'est l'amitié qui permet la communion dans la douleur et qui imprègne de douceur la main qui vient essuyer les larmes $^{28}$. Ce passage se termine par l'éloge de la «bonté » et de l'«indulgence» de Coke, qui ne s'est pas «mis en colère » contre Digby, ni n'a « eu honte » de lui, alors qu'il est lui-même connu pour sa « sagesse » et sa « constance ${ }^{29}$ ». La partie centrale de 
la lettre souligne les limites de la consolation. Digby réfute d'abord l'affirmation de Coke selon laquelle son deuil immodéré serait une manière de "rendre compte de [luimême] et de sa valeur aux yeux de Dieu et à ceux du monde ${ }^{30}$ ». C'est en raison de son tempérament, explique-t-il ensuite, marqué par un penchant à l'excès, qu'il est en proie au chagrin. Certes il essaie de mettre en pratique les exhortations de Coke, mais il n'en souffre pas moins d'une mélancolie profonde : «mon cœur est comme une table rase qui (bien que teintée de chagrin) est vierge de toute écriture ; car je n'ai ni le dessein, ni le désir de faire une chose plutôt qu'une autre ${ }^{31} »$. En guise de péroraison, enfin, Digby s'excuse de sa présomption, répond à différentes requêtes de Coke avant de terminer sa lettre par des compliments à l'intention de la femme et du fils de son protecteur.

Datée du 23 mai 1633, la troisième lettre que nous versons au dossier s'adresse à un certain Anthony Lowe ${ }^{32}$, que Digby appelle «mon ami estimé » («my worthy friend »). Plus courte (400 mots environ), elle développe les mêmes thèmes que la précédente. Dans la première partie, Digby remercie son ami de sa visite « amicale et charitable » et loue sa «tendresse " et sa "compassion ${ }^{33}$ ". Dans la deuxième partie, il reconnaît la sagesse du discours de consolation de Lowe tout en déplorant son inefficacité ; comme dans la lettre précédente, il invoque une incompatibilité de tempérament : « Je confesse que les hommes courageux doivent supporter les afflictions avec courage et magnanimité ; mais je ne suis pas de ce nombre ${ }^{34} »$. En lui prenant sa femme, affirme-til, Dieu lui a signalé la nécessité de se préparer à faire ses adieux au monde terrestre. Déjà exprimé dans l'exorde, où il avait présenté les "gestes d'humanité et de courtoisie de [ses] amis » comme " des actes de piété envers un homme mort ${ }^{35}$ ", ce désir de mort correspond à une stratégie affective fréquente dans les discours de lamentation. Dans la dernière partie de la lettre, enfin, Digby prie son ami de transmettre ses compliments à des connaissances communes; comme dans l'exorde, dominent ici les notions de service, d'obligation et de gratitude envers des personnes qui se sont montrées attentives et bien intentionnées à son égard.

12 Formant une catégorie à part dans le recueil de correspondance de Digby, ces lettres de réponse fortement codifiées correspondent à une expression routinière du discours sur les limites de la consolation, ce qui n'empêche pas la présence de certains éléments idiosyncratiques. Témoignant d'une prise en compte subtile et nuancée des problèmes inhérents à la réception du discours consolatoire, elles complexifient notre compréhension du regard que les contemporains portaient sur la consolation en tant que pratique sociale et système de valeurs.

\section{La consolation entre véhémence et bienveillance}

13 Codifié par les manuels épistolaires, le discours sur les limites de la consolation à l'œuvre dans la correspondance de Digby a des ramifications bien plus profondes. Au tournant du XVII ${ }^{\mathrm{e}}$ siècle, il trouve une caisse de résonance de premier choix dans le théâtre élisabéthain, et notamment dans les pièces de Shakespeare, où Fred B. Tromly a remarqué « la récurrence [...] de scènes où un personnage affligé résiste aux conseils donnés par un autre ${ }^{36} »$. La critique de la consolation porte dans ce cas sur les abus d'autorité éventuels que rend possible la position dominante du consolateur, détenteur d'un discours de vérité difficilement opposable, car fondé sur une normativité associant rationalité, masculinité et sagesse ${ }^{37}$. Tromly présente plusieurs cas de figure où ce sont des personnages hypocrites et manipulateurs qui s'emparent du discours de 
consolation afin d'exercer un ascendant sur des victimes affaiblies par le chagrin. Dans les lettres de réponse, la critique se situe à un autre niveau: il ne s'agit pas tant de remettre en question l'éthique du consolateur que de laisser s'exprimer le point de vue d'un destinataire réfractaire au discours de consolation. Les lettres de réponse n'en révèlent pas moins que la verticalité de la relation consolatoire est source de tensions.

La difficulté de recevoir un discours de consolation quand on est frappé par le chagrin est d'ailleurs prise en compte dans les stratégies recommandées par les manuels épistolaires et les traités de rhétorique pour contourner la réticence, voire l'hostilité, du destinataire de ce genre de discours. Érasme insiste ainsi sur la nécessité d'intervenir au bon moment, ni trop tôt, ni trop tard. Il faut faire preuve de tact et de délicatesse, éviter les plaisanteries déplacées, veiller à ne pas " paraître donner des leçons plutôt qu'une consolation amicale » et se garder de " donner des ordres, comme le bien portant aux malades ${ }^{38} »$. Une captation de bienveillance habile dans l'exorde est d'autant plus importante que les personnes affligées «détestent ceux qui les contredisent $^{39}$ ». En Angleterre, ces recommandations sont reprises par Angel Day et Thomas Wilson, les deux principaux imitateurs d'Érasme et trouvent une application directe dans les modèles de lettres de consolation inclus dans les manuels de rhétorique et d'épistolographie ${ }^{40}$. Elles reflètent une approche de la consolation qui plutôt que d'escamoter la violence potentielle induite par la verticalité de la relation entre le consolateur et le consolé, essaie d'y remédier par la mise en place de dispositifs pratiques, inspirés par une évaluation pragmatique de l'état d'esprit de celui à qui s'adresse le discours de consolation.

Les lettres de réponse mettent en évidence l'insuffisance partielle de ces stratégies de contournement. Comme nous l'avons montré plus haut, Digby proteste contre les tentatives de consolation de son entourage, imitant en cela les lettres modèles tout en les adaptant à son cas particulier. L'une des caractéristiques de cette riposte est le recours à un vocabulaire signalant la véhémence certes bien intentionnée, mais néanmoins inappropriée, du consolateur. "Il serait folie [...] de croire », lit-on dans la lettre de réponse proposée comme modèle par Angel Day, que l'on puisse "subitement» chasser le chagrin par "la véhémence de quelques discours (inspirés davantage par le zèle que par l'équité41)». Les notions de zèle («zeal») et d'équité (« equity ») sont importantes pour comprendre ce que l'on reproche ici au consolateur. Le zèle renvoie à une attitude ou à un sentiment où se rejoignent l'ardeur et l'affection ${ }^{42}$. Le terme convoque l'image d'un consolateur engagé, mû par l'amour de la cause qu'il défend, mais se laissant emporter au point de ne pas se rendre compte de la dureté excessive de son discours. Ce consolateur en oublie l'équité, c'est-à-dire la justice ou, plus précisément, la nécessité d'atténuer la rigueur de ses exigences. «Equity » était en effet un terme juridique renvoyant, selon le dictionnaire de Thomas Thomas (1587) à la «modération de la rigueur de la loi » ainsi qu'à l'«indulgence » et à la «clémence ${ }^{43}$ ». Bernard Cottret explique qu'il s'agissait d'une «branche du droit» spécifique qui «faisait appel à la "conscience" pour trancher dans un sens ou dans un autre »; il ajoute que «[1]a conscience n'est pas ici comme dans l'usage actuel un type de subjectivité ; elle est à l'inverse une forme de discernement, synonyme d'impartialité et d'objectivitée ${ }^{44}$ ». Le consolateur, défenseur par excellence de l'éthique de la modération des passions, devient donc dans la lettre de Day une figure aveuglée par le zèle. Son incapacité à prendre en compte la situation particulière de l'affligé est la conséquence d'une erreur de discernement. 
Sous la plume de Digby, la mise en cause du zèle est nettement moins virulente. Dans le corpus restreint retenu pour cette étude, le terme n'apparait qu'une seule fois, dans la lettre adressée à Anthony Lowe, que Digby remercie pour «son discours plein de réconfort, qu'il [lui] a plu de [lui] administrer avec beaucoup de sagesse et de zèle amical ${ }^{45}$ ». L'adjectif « kind » dans le syntagme « kind zeal » fait de l'ardeur de Lowe une manifestation de l'affection qu'il éprouve pour Digby. Dans le cas présent, le zèle paraît donc moins autoritaire que dans le modèle d'Angel Day. En revanche, le verbe " administer ", emprunté au vocabulaire médical, signale bien que le discours de Lowe s'inscrit dans le cadre d'une situation de communication verticale, où le consolateur, tel un médecin détenteur du savoir et de la vérité, fait prendre un remède à un patient dont il attend une attitude d'acceptation passive : dans le syntagme "administer unto me », Digby se place bel et bien en position d'objet.

17 Il convient de noter, toutefois, que dans les trois lettres que nous examinons ici, Digby adopte une attitude différenciée vis-à-vis du discours d'autorité que lui tiennent ses correspondants. Dans la lettre qu'il adresse à sa mère, il se soumet aux exhortations de celle-ci ; quand il écrit à Sir John Coke, il résiste au discours de consolation de son ami, mais s'en excuse; dans son échange avec Anthony Lowe, il rejette toute forme de réconfort sans s'en excuser. Ces différences attirent notre attention sur le fait que dans la pratique, la consolation s'exerce dans des cercles de sociabilité où les relations entre les individus sont marquées par des différences de statut et de rang plus ou moins prononcées. C'est vis-à-vis de sa mère que Digby fait preuve de la retenue la plus grande. D'une politesse exacte, sa lettre semble refléter la distance qui caractérisait les relations entre Lady Digby et son fils depuis que celui-ci avait épousé, contre la volonté maternelle, une femme admirée pour sa beauté mais dont la réputation était entachée ${ }^{46}$. Adoptant une posture d'humilité et d'obéissance, Digby signale sa volonté, perceptible également dans d'autres lettres, de revenir dans le giron familial en se soumettant à l'autorité de sa mère, qu'il décrit comme un équivalent terrestre de l'autorité divine. Une grande déférence caractérise également la lettre adressée à Sir John Coke, que Digby appelle «Right Honourable» et "Your Honour», signe de reconnaissance de sa supériorité sociale en tant que secrétaire d'État et membre du Conseil privé. S'il revendique ici son penchant à l'excès comme un élément constitutif de son tempérament, Digby atténue sa résistance à la consolation par un aveu de culpabilité et de honte: "Je confesse avec candeur que je suis excessivement fautif, mais Dieu sait que je n'y peux rien »; «Je confesse, avec assez de honte, que le chagrin s'est maintenant emparé de ma personne ${ }^{47}$ ». Il exprime par ailleurs son admiration pour Coke, qu'il représente en parfait philosophe mû par les idéaux de « sagesse » et de « constance » au point d'avoir « toujours fait preuve de la plus grande sévérité à l'égard de [lui]-même pour combattre et surmonter toutes les faiblesses humaines ». Pourtant, il a su se montrer « indulgent » à l'égard de Digby, alors qu'on aurait pu s'attendre à ce qu'il «se mette en colère ou éprouve de la honte ${ }^{48}$ ». Ces compliments relèvent certes de cette politesse dictée par les règles de civilité que l'on s'attend à trouver dans l'exorde d'une lettre de remerciements adressée à un ami et à un protecteur, mais il ne faut pas pour autant sous-estimer la force du modèle de comportement et la pression sociale qui s'en dégage. Incarnant les valeurs de rationalité, de masculinité et de sagesse qui fondent l'ethos de la consolation, Coke représente ici la norme sociale véhiculée non seulement par les manuels épistolaires mais aussi par les traités de civilité, qui exigent la retenue dans l'expression des émotions ${ }^{49}$. C'est cette norme que Digby récuse, mais avec des précautions infinies pour ne pas heurter son correspondant 
et ne pas violer les règles de la bienséance. La lettre adressée à Anthony Lowe offre à cet égard un point de comparaison intéressant. Nul sentiment de honte ni de culpabilité ne vient ici atténuer la résistance de l'épistolier à la consolation, ce qui peut s'expliquer par la différence dans la nature de sa relation avec Lowe, plus égalitaire, voire marquée par une légère supériorité de Digby, qui était chevalier ( «knight ») alors que son ami était écuyer (" esquire»).

Ces variations nous invitent à nuancer la remarque par ailleurs fort juste de Fred B. Tromly, pour qui les discours de résistance à la consolation permettent «l'ouverture d'un espace où peuvent s'exprimer conjointement la dignité et la folie d'une douleur fruste et obstinée ${ }^{50}$ ». Il est des circonstances où une telle résistance est inopportune ou ne peut se manifester qu'avec circonspection. Il n'en demeure pas moins que les manuels épistolaires, en incluant des lettres de réponse, introduisent une ouverture dialogique dans un genre plutôt caractérisé par un ethos de la fermeture, puisqu'il vise, pour reprendre une formule d'Alexandre Tarrête, « une conversion à une vérité déjà constituée ${ }^{51} »$. Les lettres de réponse constituent un espace où peuvent se confronter deux points de vue, où les valeurs de rationalité, de masculinité et de sagesse sont contrées par une série de valeurs opposées, alliant émotivité, féminité et folie. Que ce contre-discours soit lui-même fortement codifié, mobilisant notamment les normes éthiques et les dispositifs rhétoriques de la plainte, doit nous mettre en garde contre la tentation d'interpréter l'épanchement du chagrin dans les lettres de réponse comme l'expression authentique d'une émotion irrépressible et incontrôlée. Ce qu'écrit Xenia von Tippelskirch au sujet de la plainte épistolaire s'applique également aux lettres de réponse : la lamentation y est " artificielle » et cherche à « atteindre un but spécifique ${ }^{52}$ ». Dans ce sens, il est erroné de considérer ces lettres comme des " soupapes émotives ${ }^{53}$ ». Pour bien comprendre les enjeux de l'expression de la plainte dans les lettres de réponse, il faut cependant rappeler que si l'exigence de retenue était la norme, le savoir médical et la sagesse proverbiale multipliaient les mises en garde contre le refoulement du chagrin. La place prépondérante de la circulation des humeurs et des fluides dans la représentation galénique $\mathrm{du}$ corps explique que l'on ait $\mathrm{pu}$ justifier, voire recommander, l'épanchement de la tristesse de manière à provoquer une décharge émotionnelle bienfaisante. Il était communément admis, en effet, que le chagrin provoquait une contraction du cœur, un rétrécissement que l'épanchement lacrymal contrecarrait par l'évacuation d'humeurs ou de vapeurs néfastes ${ }^{54}$. Un proverbe alertait ainsi sur les dangers de la douleur refoulée : «le chagrin réprimé brise le cœur ${ }^{55}$ ». La plainte bruyante et larmoyante semblait trouver une justification dans le cri de douleur de David qui, ébranlé par la mort de son fils, voulut se retirer pour pleurer mais ne put réprimer son chagrin avant d'avoir regagné sa chambre : «David était excessivement ému " et se sentait "submergé ("overcharged») par le chagrin», écrivit en 1610 le prédicateur Robert Harris ${ }^{56}$; «son cœur allait se briser s'il ne trouvait pas le moyen d'évacuer son chagrin, c'est pourquoi il se soulagea ( discharge ») sur les escaliers, en montant, et s'écria, comme vous l'entendez, Ah, mon fils Absalon, etc. ». La mise en garde contre la douleur rentrée et la possibilité d'un soulagement temporaire au moyen d'un épanchement bref et intense, assimilé à une purgation, étaient de véritables topoi des discours sur le deuil. Cela signifie que la construction culturelle de la plainte au $\mathrm{XVI}^{\mathrm{e}}$ et au XVII ${ }^{\mathrm{e}}$ siècle intégrait l'idée d'une soupape émotive et que l'une des finalités des lettres de réponse était précisément de permettre l'expression d'une émotion intense, autorisée parce que codifiée, circonscrite et amortie grâce au recours au cadre épistolaire ${ }^{57}$. 
19 La tension dialogique induite par la confrontation de deux discours concurrents dans le cadre de ces échanges épistolaires est cependant atténuée par la nature amicale de la relation qui unit le consolateur et le consolé, une autre finalité de la lettre de réponse étant la réaffirmation des liens d'affection et d'amitié entre les correspondants. Comme le souligne Angel Day dans ses recommandations, les destinataires de lettres de consolation ont le droit de se rebiffer contre le discours qui leur est adressé, mais ils doivent toujours reconnaitre la bienveillance qui anime la démarche de leur correspondant. Digby se soumet à cette exigence dans chacune de ses lettres. Or, comme le précise Gary Schneider, « l'initiation, la consolidation et le maintien des liens sociaux » étaient une préoccupation essentielle de la pratique épistolaire des $\mathrm{XVI}^{\mathrm{e}}$ et $\mathrm{XVII}^{\mathrm{e}}$ siècles $^{58}$. L'insistance sur l'attachement mutuel qui domine l'exorde, soit environ un tiers du texte, dans les lettres que Digby écrit à John Coke et à Anthony Lowe n'était donc pas une caractéristique spécifique de la lettre de réponse. Il ne fait pourtant pas de doute que ces témoignages d'amitié infléchissent la situation de communication particulière au sein de laquelle ils s'expriment. Digby affirme que John Coke est son «meilleur ami » (« best frend»); il loue sa tendresse («the tendrest frend »), sa douceur ( gentle $)$, sa «bonté » («goodness») et sa " charité » («charity»); dans la lettre qu'il adresse à Anthony Lowe, il utilise à trois reprises l'adjectif «kind» et il met en avant l'amitié et la charité de son ami («kind and charitable visit»), son « humanité » et sa " courtoisie» (« humanitie and courtesie»), sa «tendresse » et sa "compassion» (« tendernesse and compassion »). Ce champ lexical très dense se rattache à la notion de " kindness ", dont les différentes traductions proposées par le dictionnaire d'Abel Boyer (1699) indiquent les nuances multiples : amitié, affection, bonté, douceur, humanité, bénignité, honnêteté, amitié, plaisir, faveur, grâce, bon office, service ${ }^{59}$. Comme l'a montré Linda Pollock, la notion de « kindness » était centrale dans la représentation et la pratique des relations familiales et amicales des élites éduquées. Ayant partie liée avec la civilité, elle renvoyait à des rapports qui ne relevaient pas uniquement de la politesse, mais qui étaient marqués par « une orientation positive à l'égard des autres et [par] le souci de leur bien-être ». "Kindness » décrivait des liens gouvernés par des valeurs telles que l'échange, la réciprocité et l'obligation mutuelle. Si le terme évoquait une "action" plus qu'un "sentiment", il n'en dénotait pas moins l'affection et la compassion $^{60}$. Dans la lettre qu'il adresse à John Coke, Digby décrit l'attitude compatissante de son ami philosophe comme sa seule source de réconfort: « il y a une douceur dans le chagrin lui-même quand la main d'un ami vient essuyer nos larmes ${ }^{61}$ ». Quant à Anthony Lowe, Digby utilise pour désigner son attitude pleine de commisération une expression d'origine biblique : son ami, écrit-il, «est né avec les entrailles de la tendresse et de la compassion ${ }^{62}$ ». Et quand il qualifie les "gestes d'humanité et de courtoisie de [ses] amis » d'« actes de piété envers un homme mort ${ }^{63}$ ", il mobilise la vieille notion de pietas, qui dans les textes antiques «désignait les obligations rituelles contractées envers les parents (défunts)» et qui a donné au substantif anglais piety le sens spécifique d'« amour qu'on doit avoir pour sa Patrie, \& pour ses Parents ${ }^{64} »$. Quand la rationalité du logos consolatoire s'avère inopérante, reste donc l'attachement viscéral qui unit entre eux les amis et les proches, un lien émotionnellement fort, alliant compassion, affection, souci de l'autre et sens du devoir, réaffirmé par un échange de lettres certes codifiées, mais véhiculant des valeurs essentielles pour la société anglaise de la première modernité ${ }^{65}$.

Dans le cadre des échanges épistolaires, la dynamique de la consolation naît de l'interaction féconde entre le solipsisme du deuil inconsolable et l'assurance du 
discours consolatoire. Le dédoublement du point de vue que crée la confrontation entre deux discours concurrents permet l'émergence d'une relation complexe, où la consolation ne se définit pas de façon simpliste comme l'administration d'un logos thérapeutique visant la conversion du sujet endeuillé par le déploiement d'une rhétorique délibérative. Sans nier cette dimension fondamentale de la lettre de consolation, les lettres de réponse suggèrent que la finalité de la consolation épistolaire est double : en tant que discours exhortatif ancré dans la pratique rhétorique, la lettre de consolation se charge de faire valoir la norme de la retenue émotionnelle en mettant en avant les valeurs de rationalité, de masculinité et de sagesse ; en tant que pratique sociale impliquant une interaction avec le destinataire, elle s'inscrit dans un espace où l'endeuillé peut rappeler les exigences et la temporalité propre du deuil. Il est important de comprendre qu'aucun de ces deux discours n'annule l'autre. Leur interaction permet l'expression de deux systèmes de valeurs différents, mais convergeant dans la réaffirmation commune du souci de l'autre comme fondement éthique de la vie en société.

\section{NOTES}

1. K. Digby, «A New Digby Letter-Book : "In Praise of Venetia” (continued) », The National Library of Wales Journal, vol. 9, $\mathrm{n}^{\circ}$ 4, hiver 1956 [abrégé ci-après NLWJ 9, 4], p. 446 : "Sir, I hoped to haue seene you before this time, which I pray you lett me do when your businesse and conueniency will permitt you, vnless happily you thinke you shall finde so bad company here that your Charity in visiting me will cost you too dear. " Sauf indication contraire, toutes les traductions seront les nôtres.

2. Ce recueil a été édité par V. Gabrieli : «A New Digby Letter-Book : "In Praise of Venetia" », The National Library of Wales Journal, vol. 9, n 2, hiver 1955, p. 113-148; NLWJ 9, 4, p. 440-462 ; « A New Digby Letter-Book: "In Praise of Venetia" (continued)», The National Library of Wales Journal, vol. 10, n 1, été 1957, p. 81-106; "Appendice di inediti ", dans V. Gabrieli, Un inglese italianato nell'età della Controriforma, Rome, Edizioni di storia e letteratura, 1957, p. 237-291.

3. Pour Érasme, «nul devoir ne survient plus souvent que de soulager nos amis par des paroles de consolation»: " nullum officium incidit frequentius quam vt amicorum animos consolando subleuemus » (De conscribendis epistolis, J.-Cl. Margolin éd., dans Opera omnia Desiderii Erasmi Roterdami, vol. 1, t. 2, Amsterdam, North Holland Publishing Company, 1971, p.432, LB I 426; trad. Ph. Collé et Ch. Noille: voir dans le présent numéro d'Exercices de Rhétorique, section ATELIER.)

4. Sur l'éthos de l'amitié, voir P. Trolander, Literary Sociability in Early Modern England: The Epistolary Record, Newark, University of Delaware Press, 2014, p. 77-84 ; M. Daumas, Des trésors d'amitié. De la Renaissance aux Lumières, Paris, Armand Colin, 2011, en particulier ch. 1, p. 9-40.

5. La biographie la plus récente de Digby est celle de M. Foster: "Digby, Sir Kenelm (16031665) ", Oxford Dictionary of National Biography, Oxford, Oxford University Press, 2004, éd. en ligne, janv. 2009, n. p.

6. Sur le deuil de Digby, voir C. Gittings, "Venetia's Death and Sir Kenelm's Mourning ", dans Death, Passion and Politics. Van Dyck's Portraits of Venetia Stanley and George Digby, A. Sumner dir., Londres, Dulwich Picture Gallery, 1995, p. 54-68. Les deux portraits de Digby sont reproduits ibid., p. 74 et p. 105. 
7. Sur les recueils de correspondance («letter-books»), voir J. Daybell, The Material Letter in Early Modern England. Manuscript Letters and the Culture and Practices of Letter-Writing, 1512-1635, Houndmills, Basingstoke, Hampshire, Palgrave MacMillan, 2012, p. 177-190.

8. M. Daumas, "Manuels épistolaires et identité sociale (XVI ${ }^{\mathrm{e}}-\mathrm{XVIII}{ }^{\mathrm{e}}$ siècles) », Revue d'Histoire Moderne et Contemporaine, t. 40, n 4, oct.-déc. 1993, p. 535-536. Sur les manuels épistolaires utilisés en Angleterre au XVI ${ }^{\mathrm{e}}$ et au XVII ${ }^{\mathrm{e}}$ siècle, voir Daybell, op. cit., p. 63-69.

9. A. Fleming, A Panoplie of Epistles, Londres, 1576, Short Title Catalogue [désormais STC] 11049, p. 26-29 ; A. Day, The English Secretorie, Londres, 1586, STC 6401, p. 222-223 ; M. R., A President for Young Pen-Men, Londres, 1615, STC 20584, sig. $\mathrm{G}_{2}{ }^{\mathrm{r}} ; \mathrm{J}$. Puget de la Serre, The Secretary in Fashion, tr. J. Massinger, Londres, 1640, STC 20491, p. $79 ; 80 ; 84-86$; 90-94; 99-100 (pour le texte français, voir Le secrétaire à la mode, Amsterdam, L. Elsevier, 1646).

10. Érasme, op. cit., p. 464-465 (LB I 434). On trouve bien une lettre forgée à la fin du chapitre 50 (voir dans le présent numéro d'Exercices de Rhétorique, section ATELIER, « Réponse de ma façon à une lettre de consolation ") mais elle ne comporte que deux phrases, reliées sur le mode de la pointe ingénieuse ; plus proche en cela de l'item dans un florilège, que d'une lettre à proprement parler.

11. Daybell, op. cit., p. 65 : «Book One offers a fairly comprehensive digest of letter-writing theory that synthesises Erasmian and broader humanist approaches with older traditions ». Voir Day, op. cit., p. 221.

12. Day, ibid., p. 221 : "A letter Responsorie [...] shoulde in the generall parts thereof, conteine [...] a Remuneration or friendly acceptance of their good wills, that so haue bene employed in comforting, and (if the partie so thinke meete, or the occasion so standeth) to commend the wisedome, learning, fauour, care or liking, of him that so writeth, and therein also the good effectes, the same haue wrought, if some thinges (that may be alleadged) did not impugne it, shewing his councell is farre lesse vehement then our euill, and therefore swaieth not in respect therof, in our mindes, so much as might haue done with manie others. In conclusion, that we acknowledge neuertheles his great discretion therein, and deserued accompt of hys trauell. »

13. M. R., op. cit., sig. $G_{2}{ }^{r}$ : « so farre as a man may not offend God, giue me leaue to be my selfe ».

14. Puget de la Serre, op. cit., p. 104-105; tr. Massinger, p. 93-94 (nous renvoyons au texte de l'édition française et indiquons en complément les références de la traduction anglaise).

15. Ibid., p. 101-102 ; tr. Massinger, p. 91.

16. Ibid., p. 101 ; tr. Massinger, p. 90.

17. Ibid., p. 94 ; tr. Massinger, p. 85.

18. Sur ces questions, voir F. Alazard dir., La Plainte à la Renaissance, Paris, H. Champion, 2008 ; sur la lamentation dans les traités de rhétorique, voir $\mathrm{Cl}$. Martin-Ulrich, « La lamentatio un discours sans art ? une enquête d'Érasme à Gerard Vossius ", dans Les représentations de la souffrance, actes du colloque international, université Paris-Est Créteil, des jeudi 12 mai au samedi 14 mai 2016, B. PeteyGirard et P. Séverac dir., Paris, Garnier, 2017, en cours de parution.

19. Elle compte moins de 200 mots ; voir Gabrieli, op. cit., p. 252-253.

20. Ibid. : "I humbly thanke your Ladyship for your loving and motherly letter to me which gave me as much comfort as it is possible to receive now from an earthly hand. ».

21. Ibid., p. 253 : « that we are not to sett our hears over much upon any fading object ».

22. Ibid. : " to looke upon your life since your great misfortunes and to make that a patterne to me ».

23. Ibid. : "I humbly crave your Ladyships blessing and for your hearty love that you send me, which is the greatest blessing I can have in this worlde, I returne the humble and unfayned duty of Your Ladyships most humble and most obedient sonne. "

24. Comme beaucoup de catholiques briguant des fonctions publiques, Digby s'était converti à l'Église anglicane ; après la mort de sa femme il entama toutefois un processus de reconversion qu'il rendit public en 1634. Il est possible de lire son recueil de correspondance comme un ensemble biographique retraçant son parcours spirituel: voir M. B. Young, "Coke, Sir John [1563-1644] », Oxford Dictionary of National Biography, Oxford, Oxford University Press, 2004, éd. en 
ligne janv. 2016, n. p. ; Foster : «Digby, Sir Kenelm [1603-1665] », op. cit., n. p. ; P. Barros, « Deuil, mélancolie et conversion : errances et itinérances spirituelles dans les lettres de deuil de sir Kenelm Digby ", dans Itinérances spirituelles : écriture et mise en récit du voyage intérieur (XV'-XVIII ${ }^{e}$ siècles), I. Kirschleger et F. Poulet dir., Paris, H. Champion, à paraître.

25. Gabrieli, op. cit., p. 254-255.

26. Ibid., p. $254:$ : you are the best frend and the wisest man that ever I knew ».

27. Ibid. : "They tell one barely what he should do, but they can scarcely prepare ones affections before hand to digest their praeceptes."

28. Ibid. : "There is a kinde of sweetenesse in sorrow itselfe, when ones teares are wiped away by a frendes hande."

29. Ibid. : "I know your wisedome and constancy to be such as I might rather have expected that you would have been angry with me or ashamed of me, then that you would have commiserated me. [...] this is the effect of a transcendent goodnesse in you that though you are, and ever have bin, most severe to your selfe in resisting and overcoming all thinges of humane frailty, yet you can therein be gentle and indulgent to your frendes. »

30. Ibid.: " that my complaint proceedeth from a noble and earnest desire to give accompte of Myself and worth both to God and the world».

31. Ibid., p. 255 : «my heart is like a tabula rasa in which (though it be tincted over with sorrow) nothing is written; for I have neither designe nor desire to any one thing more then an other. "

32. NLWJ 9, 4, p. 449.

33. Ibid.: "giue you abliged thankes for your late kind and charitable visite to me"; "you who were borne with bowells of tendernesse and compassion $»$.

34. Ibid. : «I confesse, afflictions are to be borne by braue men with constancy and magnanimity: but I am not of that number».

35. Ibid. : « all humanity and courtesie of my frendes towardes me may more properly be called piety to a dead man ».

36. F. B. Tromly, "Grief, Authority and the Resistance to Consolation in Shakespeare ", dans Speaking Grief in English Literary Culture: Shakespeare to Milton, éd. M. Swiss et D. A. Kent, Pittsburgh, Duquesne UP, 2002, p. $21:$ "[T]he surprisingly frequent recurrence of scenes in which a character who is grieving resists the counsel that another has proffered. »

37. Ibid., p. 25-26.

38. Érasme, op.cit., p. 432-435 (trad. Ph. Collé et Ch. Noille: voir dans le présent numéro d' Exercices de Rhétorique, section ATELIER). La citation est extraite de la p. 435 (LB I 427) : « cavendum ne non tam amice consolari quam praecipere videamur [...] ne tanquam sani aegrotantibus imperemus ».

39. Ibid., p. 433 (LB I 426) : «Oderunt dissentientes ».

40. Day, op. cit., p. 211-217; Th. Wilson, The Arte of Rhetorique, Londres, 1560, STC 25800, fol. $34^{\mathrm{r}-} 44^{\mathrm{r}}$.

41. Day, op. cit., p. 222 : « Follie were it for mee to thinke or you to beleeue, that the pensiue imagination of a thing so neere [...] coulde with the vehemencie of a fewe specches (more of zeale then equitie deliuered) be sodenly remooued."

42. Voir par exemple la définition donnée par le dictionnaire bilingue d'A. Boyer : "affection ardente, Ardeur » (The Royal Dictionary, Londres, 1699, Wing B3917, n. p.).

43. Th. Thomas, Dictionarium Linguae Latinae et Anglicanae, Londres, 1587, STC 24008, sig. $\mathrm{B}_{6}^{\mathrm{v}}$ : « moderation of rigour of the lawe: a thing which is of right and reason : steadfastnes of minde, which will be troubled with nothing : mildenes and clemencie. "

44. B. Cottret, Thomas More, Paris, Tallandier, 2012, p. 363.

45. NLWJ 9, 4, p. 449 : « such comfortable discourse as (I thanke you), with much wisedom and kind zeale, you were pleased to administer vnto me ». 
46. Une rumeur courait selon laquelle Venetia Digby avait eu des relations avec plusieurs hommes avant son mariage (voir J. Aubrey, Brief Lives, R. Barker éd., Woodbridge, The Boydell Press, 2004, p. 105).

47. Gabrieli, op. cit., p. 254 et 255 : « For I confesse ingenuously I am much to blame, but God knoweth I can not helpe it »; «I confess, with shame enough, my sorrow mastereth me now ».

48. Voir note 29.

49. Voir à ce sujet B. Capp, qui montre que dans la pratique aussi, les hommes se retenaient le plus souvent de pleurer («"Jesus Wept” But Did the Englishman? Masculinity and Emotion in Early Modern England », Past \& Present, $n^{\circ} 224$, p. 75-108).

50. Tromly, op. cit., p. 22 : « the dignity and folly of unyielding, untutored sorrow ».

51. Cette remarque concernant plus spécifiquement le dialogue de consolation s'applique a fortiori à la lettre de consolation. Voir A. Tarrête, «Remarques sur le genre du dialogue de consolation à la Renaissance ", Renaissance, Humanisme, Réforme, n 57, déc. 2003, p. 136.

52. X. von Tippelskirch, "Se plaindre par lettre (en Italie au XVI ${ }^{\mathrm{e}}$ et au début du XVII ${ }^{\mathrm{e}}$ siècle) ", dans F. Alazard dir., La Plainte à la Renaissance, op. cit., 2008, p. 324. Voir aussi les remarques de C. Lignereux dans "Une routine de la civilité épistolaire: l'expression de la condoléance", Exercices de rhétorique, $\mathrm{n}^{\circ}$ 6, 2016, n. p., URL : http:// rhetorique.revues.org/437 ; DOI : 10.4000/ rhetorique. 437

53. Tippelskirch, op. cit., p. 324.

54. Voir par exemple T. Bright, A Treatise of Melancholie, Londres, 1586, STC 3747, p. 161.

55. "Grief pent up will break the heart " (M. P. Tilley, A Dictionary of the Proverbs in England in the Sixteenth and Seventeenth Centuries, Ann Arbor, The University of Michigan Press, 1950, proverbe $\left.\mathrm{n}^{\circ} \mathrm{G} 449\right)$.

56. R. Harris, Absaloms Funerall, Londres, 1610, STC 12817, p. 1-2: « his heart would breake, unlesse it found a vent, and therefore he discharges himselfe at the stayres, as he goes, and breakes forth, as you heare, 0 my sonne Absalom, \&c. » Voir 2 Sm 18, 33.

57. Voir G. Schneider, The Culture of Epistolarity. Vernacular Letters and Letter Writing in Early Modern England, 1500-1700, Newark, University of Delaware Press, 2005: pour G. Schneider, les lettres avaient une fonction sociale de "tampon " qu'on utilisait "dans des situations difficiles ou chargées en émotions" dans la mesure où elles permettaient "l'expression textuelle » d'émotions difficilement communicables dans des situations de «face-à-face " (op. cit., p. 132-133 : «It is crucial to examine the letter's function as a "social buffer" employed in difficult or emotionally charged situations [...]»; " the letter [...] served as a suitable social "container" for emotions more appropriately textualized rather than expressed face to face in a society more intent on decorum and on the regulation of emotion $»)$.

58. Schneider, op. cit., p. 67 : «the implication of much of the language of letter-writing is that even if letters said nothing about news or business, they spoke volumes about connection, about the initiation, consolidation, and maintenance of social ties ».

59. Boyer, op. cit., n. p.

60. L. Pollock, "The Practice of Kindness in Early Modern Elite Society ", Past \& Present, $n^{\circ} 211$, mai 2011, en particulier p. 124, 127 (pour la citation), 131, 136, 140-142 ; pour la citation, voir p. 127 : « Kindness described a positive orientation towards other people, and a concern for their welfare ».

61. Voir note 28.

62. $1 \mathrm{Jn} 3,17$; voir note 33 .

63. Voir note 35.

64. M. Lauwers, La Mémoire des ancêtres, le souci des morts. Morts, rites et société au Moyen Âge (Diocèse de Liège, XI ${ }^{e}$-XIII ${ }^{e}$ siècles), Paris, Beauchesne, 1997, p. 196 ; Boyer, op.cit., n. p. Voir aussi la définition de pietas dans le dictionnaire de Th. Thomas (op. cit., sig. $\mathrm{Xx}_{8}{ }^{\mathrm{r}}$ ): «The dutie, honestie, conscience, good dealing, that is due first to God, and afterward to our fathers and mothers, children, 
kinsfolke, and vncle: deuotion, godlines, godly affection : naturall and reuerent loue, naturall affection or zeale, reuerence, remorse of conscience. "

65. Sur la place de la compassion dans la pratique de la consolation à l'époque moderne, voir " "Hee Left Them Not Comfortlesse by the Way": Grief, Compassion and Consolation in Early Modern English Culture », dans K. Ibbett et K. Steenbergh dir., Compassion in Early Modern Culture, Oxford, Oxford University Press, à paraître.

\section{AUTEUR}

\section{PAULA BARROS}

Université Paul-Valéry Montpellier 3 - IRCL, UMR 5186 